\title{
Capacidades Dinâmicas, Dinamismo Ambiental e Vantagem Competitiva: Evidências de uma Instituição Comunitária de Ensino Superior
}

\author{
Dynamic Capabilities, Environmental Dynamism and Competitive Advantage: Evidence \\ from a Community Higher Education Institution
}

\section{Capacidades Dinámicas, Dinamismo Ambiental y Ventaja Competitiva: Evidencia de una Institución Comunitaria de Educación Superior}

Iane Emanuelle Medeiros Gomes de Brito
ianegomes @ unochapeco.edu.br
http://lattes.cnpq.br/1590926393040957
https://orcid.org/0000-0002-6966-2438

Givanildo Silva ${ }^{1}$

givanildo.silva@unochapeco.edu.br http://lattes.cnpq.br/6693950622636292 https://orcid.org/0000-0002-9328-1563

Rodrigo Barichello ${ }^{1}$ rodrigo.b@unochapeco.edu.br http://lattes.cnpq.br/6760700105264006 https://orcid.org/0000-0002-0358-1467

Cristian Rebonatto ${ }^{1}$ cristianrebonatto@unochapeco.edu.br http://lattes.cnpq.br/7137268558968612 https://orcid.org/0000-0002-4103-9169

Recebido em: 30/03/2021 / Revisão: 20/04/2021 / Aprovado em: 01/06/2021

Editores responsáveis: Prof. Dr. Antônio Giovanni Figliuolo Uchôa e Prof. Dr. Jonas Fernando Petry 
Capacidades Dinâmicas, Dinamismo Ambiental e Vantagem Competitiva: Evidências de uma Instituição Comunitária de Ensino Superior

\title{
Resumo
}

Este artigo se propõe a relacionar as capacidades dinâmicas, dinamismo ambiental e vantagem competitiva. A relevância do tema se dá pela evidenciação de critérios fundamentais para o desempenho de uma organização e o aumento cada vez maior de mudanças rápidas e assertivas nas estratégias empresariais, para que uma organização possua efetividade em sua gestão. Este estudo teve como amostra de pesquisa, gestores de uma universidade comunitária e os dados foram coletados por meio de questionário enviado por endereço eletrônico a partir do setor de recursos humanos. Foram obtidas 20 respostas, representando $20 \%$ do número total de respondentes. A análise dos dados foi descritiva, mostrando que uma instituição de ensino superior necessita de senso de capacidades estratégicas, capacidade de decisão antecipada, capacidade de alterar a implementação das estratégias, vantagem competitiva em relação aos seus concorrentes e percepção de dinamismo ambiental, para que conquiste níveis elevados de sustentação na gestão estratégica.

Palavras-Chave: Capacidades Dinâmicas. Dinamismo ambiental. Vantagem Competitiva. Estratégia Empresarial.

\section{Dynamic Capabilities, Environmental Dynamism and Competitive Advantage: Evidence from a Community Higher Education Institution}

\begin{abstract}
This article proposes to relate dynamic capabilities, environmental dynamism and competitive advantage. The relevance of the theme is due to the disclosure of fundamental criteria for the performance of an organization and the increasing increase of rapid and assertive changes in business strategies, so that an organization has effectiveness in its management. This study had as a research sample, managers from a community university and the data were collected through a questionnaire sent by email from the human resources sector. Twenty responses were obtained, representing $20 \%$ of the total number of respondents. The analysis of the data was descriptive, showing that a higher education institution needs a sense of strategic capabilities, the ability to make early decisions, the ability to change the implementation of strategies, competitive advantage in relation to its competitors and perception of environmental dynamism, in order to achieve high levels of support in strategic management.
\end{abstract}

Keywords: Dynamic Capabilities. Environmental dynamism. Competitive advantage. Business strategy.

\section{Capacidades Dinámicas, Dinamismo Ambiental y Ventaja Competitiva: Evidencia de una Institución Comunitaria de Educación Superior}

\section{Resumen}

Este artículo propone relacionar capacidades dinámicas, dinamismo ambiental y ventaja competitiva. La relevancia del tema se debe a la divulgación de criterios fundamentales para el desempeño de una organización y al incremento creciente de cambios rápidos y asertivos en las estrategias de negocio, para que una organización tenga efectividad en su gestión. Este estudio tuvo como muestra de investigación, administradores de una universidad comunitaria y los datos fueron recolectados a través de un cuestionario enviado por correo electrónico desde el sector de recursos humanos. Se obtuvieron veinte respuestas, lo que representa el $20 \%$ del 
número total de encuestados. El análisis de los datos fue descriptivo, mostrando que una institución de educación superior necesita un sentido de capacidades estratégicas, la capacidad de tomar decisiones tempranas, la capacidad de cambiar la implementación de estrategias, la ventaja competitiva en relación con sus competidores y la percepción de dinamismo ambiental, con el fin de lograr altos niveles de apoyo en la gestión estratégica.

Palabras clave: Capacidades dinámicas. Dinamismo ambiental. Ventaja competitiva. Estrategia empresarial. 
Capacidades Dinâmicas, Dinamismo Ambiental e Vantagem Competitiva: Evidências de uma Instituição Comunitária de Ensino Superior

\section{INTRODUÇÃO}

O conceito de estratégia aplicada aos estudos de negócios só aparece com grande frequência a partir de 1960 (Ansoff, 1965; Chandler, 1962). Por isso, contextos das organizações empresariais, a estratégia às vezes é definida com certo grau de oportunismo. Sua definição geralmente permanece implícita, aberta à interpretação intuitiva (Hakasson, 2006). O conteúdo atribuído ao conceito varia de um autor para outro, mas a essência de muitas definições converge no conceito de estratégia como o padrão no fluxo de decisões e atividades que caracteriza a partida que uma organização consegue com seu ambiente, e que é determinante para a consecução de seus objetivos (Mintzberg \& Mchugh, 1985; Hofer \& Schendel, 1980).

Os desenvolvimentos na economia global mudaram o equilíbrio tradicional entre cliente e fornecedores, agora as empresas precisam ser mais centradas no cliente, especialmente porque a tecnologia evoluiu para permitir o fornecimento de informações e soluções para clientes com menor custo (Teece, 2010). Para que uma empresa possua vantagem competitiva é necessário estabelecer uma estratégia que agregue valor sob certas condições, para impor um limite superior ao quanto a empresa pode capturar (Brandenburger, 1996). Portanto, a estratégia de negócios define o plano de longo prazo de ação que uma empresa pode seguir para atingir metas (Zahra \& Covin, 1993).

Teece (2010) enfatiza que o novo ambiente na economia global também ampliou a necessidade de considerar não apenas como atender às necessidades dos clientes de maneira mais astuta, mas também como capturar valor ao fornecer novos produtos e serviços. Sendo assim, as organizações devem investir com confiança no desenvolvimento de capacidades dinâmicas para lidar com mudanças ambientais, evitando rigidez e armadilhas essenciais ( $\mathrm{Li} \& \mathrm{Liu}, 2014)$. Um modelo de negócios articula a lógica e fornece dados e outras evidências que demonstram como uma empresa cria e agrega valor aos clientes (Teece, 2010).

Portanto, a estratégia de negócios empresariais deve centrar-se na agregação de valor para os clientes, proporcionando a experiência de compra e de fidelização. No intuito de estudar a respeito do tema, percebeu-se oportunidade de desenvolver um estudo de caso em uma instituição de ensino superior com viés comunitário. Na literatura sobre universidades, o processo de tornar-se uma organização reflete uma dinâmica de racionalização mais ampla, obrigando a universidade a adquirir as características de uma entidade organizacional integrada e de atuação estratégica (Ramirez, 2006). Tal afirmação suscita a seguinte questão: qual a relação entre capacidades dinâmicas, dinamismo ambiental e vantagem competitiva em uma instituição de ensino superior?

Os diferentes elementos que precisam ser determinados no design do modelo de negócios estão listados na Figura 1. 
Capacidades Dinâmicas, Dinamismo Ambiental e Vantagem Competitiva: Evidências de uma Instituição Comunitária de Ensino Superior

Criar valor para os clientes, atrair pagamentos e converter pagamentos

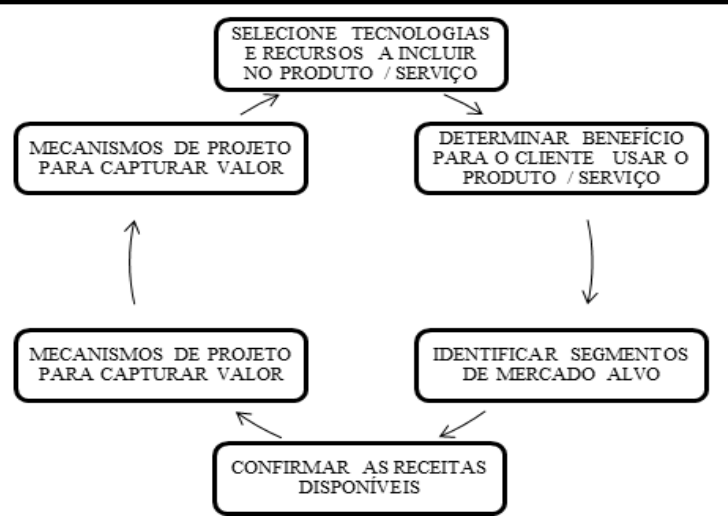

Figura 1: Elementos de design para modelo de negócios (Teece, 2010).

Visando responder adequadamente ao questionamento proposto, estabeleceu-se como objetivo do estudo avaliar a relação entre capacidades dinâmicas, dinamismo ambiental e vantagem competitiva, por meio de coleta de dados quantitativa e análise descritiva. Partindo deste objetivo espera-se que o estudo possa contribuir tanto pragmática quanto teoricamente. Do ponto de vista teórico, almeja-se contribuir para diminuir a lacuna de pesquisas que abordam este tema.

\section{REVISÃO DA LITERATURA}

As próximas seções discorrem acerca dos conceitos necessários à adequada compreensão do texto e que servirão como base de sustentação do arcabouço teórico da pesquisa.

\subsection{Capacidades Dinâmicas}

O conceito de capacidades dinâmicas propõe a visão de que as empresas que possuem uma capacidade de alta ordem são consideradas capazes de criar, estender ou modificar propositalmente sua base de conhecimento para responder às mudanças em um ambiente de negócios (Eisenhardt, 2000; Helfat 2009). Portanto, a dinâmica de implementação molda as capacidades de formulação de estratégias (Thoenig, 2016). Tregoe \& Zimmerman (1980), relatam que a palavra estratégia foi adicionada ao universo dos negócios, sendo utilizada pelas organizações, significando a forma pela qual as empresas se desempenhavam e agiam diante dos seus concorrentes. Segundo Porter (1999), estratégia é obter uma posição muito preciosa e exclusiva, criando um grupo distinto de ações, por meio de determinadas escolhas para se obter um melhor ganho em relação à concorrência.

O uso do termo estratégia, geralmente enfatiza o papel e a importância do pessoal de alto nível, como presidentes, reitores, vice-reitores e presidentes de conselho em uma instituição de ensino superior (Thoenig, 2016). Para Oliveira (2001), a ação estratégica remete-se ao mundo do planejamento estratégico, bastante utilizado na vida das empresas. A ação estratégica interage com os objetivos de longo prazo e com as formas de alcançá-los. Sendo assim, espera-se que os estrategistas, a fim de serem bem-sucedidos, forneçam insumos e produzam resultados, que sejam perspicazes, mas flexíveis, que tenham controles rígidos em algumas áreas da empresa e 
Capacidades Dinâmicas, Dinamismo Ambiental e Vantagem Competitiva: Evidências de uma Instituição Comunitária de Ensino Superior

menos controle em outras, que tenham uma visão ampla inspiradora e atenção meticulosa aos detalhes (Moore, 1995).

O conceito de capacidade estratégica descreve como uma instituição alinha seus componentes internos para atingir objetivos comuns. Refere-se a um processo orientado à ação coletiva e contínua (Thoenig, 2016). A modificação estratégica mostra-se como um fato essencial na análise dos processos de adaptação organizacional, sobrevivência e crescimento exponencial das organizações (Sausen, 2002). A extensão em que as prioridades, preferências e iniciativas tomadas dentro da instituição ao longo do tempo e em seu ambiente se harmonizam depende muito de quão bem a linha programática que a organização cria se encaixa, assim, organizando recursos adequados para serem alocados para esses fins (Thoenig, 2016).

Frølich (2019) enfatiza que capacidade estratégica é um conceito que se relaciona com os processos associados a formação, a organização e a implementação de estratégias em universidades e em faculdades. Em termos concretos, capacidade estratégica refere-se à maneira como todas as diferentes partes interessadas, com suas próprias aptidões e agendas de trabalho, se tornam compatíveis entre si, adaptando suas iniciativas de acordo com as decisões tomadas na organização (Thoenig, 2016).

A força das capacidades dinâmicas de uma empresa é vital de várias maneiras para sua capacidade de manter a lucratividade a longo prazo, incluindo a capacidade de projetar e ajustar modelos de negócio (Teece, 2018). As organizações que se aproximam muito das formas tradicionais de operação serão prejudicadas em sua capacidade de obter sucesso. Por outro lado, aqueles que se concentram no desenvolvimento de novos produtos e processos, juntamente com a inovação do modelo de negócios, alavancam seus recursos dinâmicos (Schoemaker et al., 2018). Portanto, as capacidades dinâmicas melhoram a agilidade organizacional e o desempenho organizacional (Wamba et al., 2020).

\subsection{Dinamismo Ambiental}

Teece, Pisano e Shuen (1997) propõem que as empresas devem constantemente adaptar, reconfigurar e renovar seus recursos e suas capacidades para abordar mudanças ambientais, que é o conceito universal de capacidades dinâmicas. Jansen (2006) ressalta que o dinamismo ambiental é caracterizado por mudanças tecnológicas, variações nas preferências do cliente, mudanças na demanda de produtos e a imprevisibilidade da mudança. Devido ao alto índice de mudança no ambiente que estão enfrentando, as organizações geralmente têm recursos muito limitados de informações e, portanto, previsões ruins em termos do que levará ao sucesso (Hou et al., 2019).

Esses tipos de ambientes dinâmicos existem nos mercados ao redor do mundo, nas economias desenvolvidas e em desenvolvimento (Story et al., 2015). O dinamismo ambiental força as organizações a adotar e implementar procedimentos e regras escritas para evitar erros, onde ambas as variáveis ambientais aumentam os graus de formalização e centralização da organização (Aslan, 2021).

O dinamismo ambiental também pode ser considerado imprecisão externa e pode ser definido como a taxa de mudança ou imprevisibilidade predominante no ambiente de um negócio (Eroglu \& Hofer, 2014). O dinamismo ambiental fornece uma oportunidade para a melhoria dos processos existentes e ou o desenvolvimento de novos produtos e processos (Chan et al., 2016). Pesquisadores do empreendedorismo argumentam que ambientes dinâmicos geralmente 
Capacidades Dinâmicas, Dinamismo Ambiental e Vantagem Competitiva: Evidências de uma Instituição Comunitária de Ensino Superior

favorecem empresas empreendedoras devido à sua capacidade de se adaptar rapidamente e responder a mudanças decorrentes das demandas de clientes, concorrentes e avanços tecnológicos (Covin \& Slevin, 1991; D’aveni, 1994; Zahra, 1993). Conforme Zhang (2021), o dinamismo ambiental desempenha um papel considerável em determinar se as empresas adotarão a inovação de processos verdes.

Em seus estudos, Fran (2017), enfatiza que quanto maior o dinamismo ambiental na empresa, mais predominantes e importantes são as capacidades dinâmicas, e que a busca pelo aprendizado e flexibilidade são maiores quando o dinamismo ambiental é alto. Além disto, o dinamismo ambiental também favorece a formação da cooperação em pesquisa e desenvolvimento nas organizações (Wang, 2020). Chirico e Bau (2014), relatam que o dinamismo ambiental é uma noção, relativa à incerteza, complexidade e mudanças imprevisíveis no ambiente em que as empresas estão envolvidas. Zhang e Zhu (2021) corroboram que o dinamismo ambiental tem influência positiva e significativa na flexibilidade estratégica de uma empresa.

\subsection{Vantagem Competitiva}

A literatura existente percebeu que as empresas podem obter vantagem competitiva e desempenho superior usando recursos de maneira eficiente e inovadora (Amwar, 2018; Demil et al., 2015; Shane \& Venkataraman, 2000). A teoria sobre dinâmica competitiva conceitua a estratégia como um conjunto de ações utilizadas pelas empresas para melhorar suas posições de mercado (Ferrier, Smith \& Grimm, 1999; Yu \& Cannella Jr., 2007). Uma ação estratégica é definida como uma ação competitiva dirigida, específica e observável, iniciada por uma empresa para melhorar sua posição competitiva relativa (Smith, Ferrier \& Ndofor, 2001). Rothaermel (2016) relata que ganhar e sustentar vantagem competitiva são as questões definidoras da estratégia. Possas (1999) afirma que a competitividade pode ser mensurada por indicadores de desempenho ex-ante e ex-post. Os indicadores ex-ante estão ligados às formulações estratégicas para desenvolver vantagens competitivas sustentáveis e tem como objetivo o acesso privilegiado a fornecedores, qualificação e formação de mão de obra, diversificação e diferenciação de produtos, inovações tecnológicas, entre outros.

A vantagem competitiva das instituições de ensino decorre do impacto sobre o nível de cada aluno, funcionário e da própria instituição (Al Shobaki \& Abu-Naser, 2017). Em países emergentes, como o Brasil, a flexibilidade e a velocidade das ações estratégicas podem ser valiosas para se obter vantagem competitiva. Quanto mais volátil for o ambiente institucional, mais crítica é a velocidade de ação como um parâmetro competitivo (Chang \& Park, 2012; Gadiesh, Leung \& Vestring, 2007). No entanto, apesar da importância da velocidade competitiva em tais contextos, poucos pesquisadores investigaram a dinâmica competitiva em economias emergentes (Cui, Meyer \& Hu, 2014). Com maior fragmentação de mercados e clientes mais exigentes, prestadores de serviços estão mudando o foco para fornecer soluções inovadoras e focadas no cliente, projetadas para oferecer maior valor ao cliente e vantagem competitiva sustentada (Salunke, 2019).

Uma empresa que supera seus concorrentes tem uma vantagem competitiva. Se esta empresa é capaz de dominar seus concorrentes por períodos prolongados, diz-se que a empresa possui uma vantagem competitiva sustentada (Rothaermel, 2016). Salunke et al. (2019), argumenta que as capacidades dinâmicas, ao disponibilizar novas configurações de recursos de conhecimento, permitem que as empresas busquem soluções inovadoras focadas no cliente e, por sua vez, obtenham vantagem competitiva. As empresas estão adotando cada vez mais uma perspectiva 
Capacidades Dinâmicas, Dinamismo Ambiental e Vantagem Competitiva: Evidências de uma Instituição Comunitária de Ensino Superior

orientada para o cliente como fonte de vantagem competitiva, integrando ofertas de serviços nos processos de negócios dos clientes (Matthyssens \& Vandenbempt, 2008). Existe uma forte correlação entre o nível de exercício de estratégias de excelência na educação e a conquista de instituições de ensino superior para a vantagem competitiva sustentável (Al Shobaki \& AbuNaser, 2017).

Nos tempos modernos, chegou-se à conclusão de que a aquisição e o uso efetivo de conhecimento é a única maneira de obter uma vantagem competitiva sustentável no mercado (Mahdi et al., 2019). Chiavenato (2010) afirma que o universo corporativo requer líderes para a condução bem-sucedida das empresas e a liderança retrata a maneira mais eficiente de incentivar e de renovar as organizações e impulsioná-las rumo ao sucesso e à competitividade. Conforme Borges-Andrade (2006), a competência é vista como o conjunto de conhecimento, habilidade e atitude que são necessários para exercer uma atividade, bem como, os comportamentos adotados no trabalho.

Com efeito, de acordo com Coutinho e Ferraz (1994), o êxito competitivo de um setor de atividade está expressamente vinculada com a dinâmica voltada para o crescimento e renovação das capacidades tecnológicas que proporcionam vantagens competitivas capazes de mantê-las no mercado. A capacidade tecnológica também é a união de recursos de natureza cognitiva e intangível, mas capazes de explicar seu desempenho no mercado; tendo como base, as organizações são capazes de executar atividades de produção e de inovação (Figueiredo, 2009).

Empresas com uma vantagem competitiva mais forte e eficaz produzem maior produtividade (Lechner \& Gudmundsson, 2014). Portanto, uma empresa que desfruta de uma vantagem competitiva não apenas é mais lucrativa que seus concorrentes, mas também cresce mais rápido porque é capaz de capturar mais participação de mercado, diretamente dos concorrentes ou do crescimento geral do setor, devido à maior competitividade da empresa (Rothaermel, 2016). As organizações perceberam que o conhecimento, seu uso efetivo e a rápida aquisição e utilização de novos conhecimentos representam a única fonte de vantagem competitiva sustentável (Schiuma et al., 2012). Sendo assim, o conhecimento processado nas instituições educacionais auxilia a decidir a direção que seguirão no futuro e quão competitivos serão no mundo dos negócios (Mahdi et al., 2019).

Ativos tangíveis não são mais considerados a base da vantagem competitiva, pois na comunidade empresarial moderna, é mais significativo atrair capital intelectual (Halawi et al., 2005). Assim, a magnitude da vantagem competitiva de uma empresa é a diferença entre o valor percebido criado e os custos para produzir o bem ou serviço em comparação com seus concorrentes diretos (Rothaermel, 2016). Para que haja uma vantagem competitiva em uma organização, ela precisa produzir valor econômico adicional em seu mercado de produtos do que o concorrente (Peteraf \& Barney, 2003). No presente, as organizações precisam se concentrar em alcançar uma posição de vantagem competitiva aumentando o desempenho da organização (Raduan et al., 2009). Desta forma, para criar e sustentar vantagem competitiva, os gerentes da empresa devem entender os pontos fortes e fracos da empresa, bem como suas oportunidades e ameaças que se apresentam no ambiente externo da empresa (Rothaermel, 2016).

\section{MATERIAIS E MÉTODOS}

Na elaboração deste estudo foi utilizado o método de coleta de dados quantitativo, que é "tradicionalmente obtido por meio de várias escalas numéricas e são usadas quando o 
Capacidades Dinâmicas, Dinamismo Ambiental e Vantagem Competitiva: Evidências de uma Instituição Comunitária de Ensino Superior

pesquisador está utilizando modelos teóricos e problemas de pesquisa bem definidos". Para utilização deste método aplicou-se questionário e em seguida a análise dos dados coletados (Hair Jr., 2005 p. 152).

O método para a análise deste estudo caracteriza-se como descritivo, pois busca características de uma população a fim de identificar relações entre variáveis, permitindo conhecimentos válidos e verdadeiros, identificando erros e auxiliando as decisões do pesquisador (Lakatos \& Marconi, 2003).

Quanto à abordagem, classifica-se como quantitativa, que para Richardson (2014, p. 70), "como o próprio nome indica, caracteriza-se pelo emprego de quantificação tanto nas modalidades de coleta de informações, quanto no tratamento delas por meio de técnicas estatísticas, desde as mais simples às mais complexas". Em relação aos procedimentos, classifica-se como levantamento, por meio da aplicação de questionário. O levantamento caracteriza-se pela interrogação direta das pessoas cujo comportamento se deseja conhecer (Martins, 2002). Neste caso, o levantamento foi realizado a partir de um questionário específico, fundamentado no estudo de Li \& Liu (2014), com adequações ao contexto universitário.

Para efetuar o pré-teste, o questionário foi enviado à diretora de desenvolvimento humano da universidade, com o objetivo de validar as adaptações efetuadas a partir do estudo utilizado como base. Em seguida, o questionário foi enviado aos 100 gestores da instituição por meio de correio eletrônico, acompanhado de texto explicativo sobre o objetivo da pesquisa, finalidade dos dados e assegurando a confidencialidade das respostas. Para o envio do questionário, foi encaminhado um e-mail pela Diretoria de Desenvolvimento Humano da instituição que continha o link eletrônico para acesso ao questionário e foi elaborado e disponibilizado via ferramenta Google Docs. Com isso, foram obtidas 20 respostas, representando $20 \%$ do número total de respondentes.

Foi utilizada escala Likert de 5 pontos, de discordo totalmente a concordo totalmente. Os dados coletados foram analisados por meio de análise descritiva, utilizando as ferramentas estatísticas de média e mediana para interpretação dos indicadores.

\section{RESULTADOS}

Para analisar os dados coletados, utilizou-se o cálculo da média e mediana de cada variável apresentada na pesquisa. Nas tabelas a seguir, são apresentados os resultados obtidos.

Tabela 1: Senso de Capacidade Estratégica

\begin{tabular}{|c|c|c|}
\hline \multicolumn{1}{|c|}{ Variáveis } & Média & Mediana \\
\hline Nós conseguimos perceber a mudança ambiental antes dos concorrentes - SCE1 & $\mathbf{3 , 1 5 8}$ & $\mathbf{3}$ \\
\hline Muitas vezes temos reuniões para discutir a demanda do mercado. SCE2 & $\mathbf{3 , 5 0 0}$ & $\mathbf{3 , 5}$ \\
\hline Conseguimos entender completamente o impacto do ambiente interno e externo. SCE3 & $\mathbf{2 , 8 9 5}$ & $\mathbf{3}$ \\
\hline Conseguimos perceber as grandes oportunidades e potenciais ameaças. SCE4 & $\mathbf{3 , 4 2 1}$ & $\mathbf{4}$ \\
\hline Nossa instituição possui um sistema de gerenciamento de informações muito bom. SCE5 & $\mathbf{2 , 9 4 7}$ & $\mathbf{3}$ \\
\hline Temos boa capacidade de observação e julgamento. SCE6 & $\mathbf{3 , 2 7 8}$ & $\mathbf{3}$ \\
\hline
\end{tabular}

Fonte: Dados da pesquisa (2019).

UFAMBR, Manaus, v. 3, n. 1, art. 3, pp. 39-53, janeiro-junho, 2021 http://www.periodicos.ufam.edu.br/ufambr 
Capacidades Dinâmicas, Dinamismo Ambiental e Vantagem Competitiva: Evidências de uma Instituição Comunitária de Ensino Superior

Observa-se que, a percepção de mudança ambiental antes dos concorrentes, a frequência de reuniões para discutir a demanda do mercado, a percepção de grandes oportunidades e potenciais ameaças, e a boa capacidade de observação e julgamento, obtiveram índices entre 3,158 e 3,500 e ressalta que, para os gestores da instituição, têm-se essas variáveis bem aplicadas em suas rotinas de gestão estratégica. Vários estudiosos argumentam que ambientes dinâmicos geralmente favorecem empresas empreendedoras devido à sua capacidade de se adaptar rapidamente e responder a mudanças decorrentes das demandas de clientes, concorrentes e avanços tecnológicos (Covin \& Slevin, 1991; D’aveni, 1994; Zahra, 1993).

Em relação a conseguir entender completamente o impacto do ambiente interno e externo, e possuir um sistema de gerenciamento de informações muito bom, tem-se indicadores entre 2,895 e 2,947, que indicam a necessidade de melhorias. Portanto, devido ao alto índice de mudança no ambiente que estão enfrentando, as organizações geralmente têm recursos muito limitados de informações e, portanto, previsões ruins em termos do que levará ao sucesso (Hou et al., 2019).

Tabela 2: Capacidade de Decisão Antecipada

\begin{tabular}{l|c|c}
\hline \multicolumn{1}{c|}{ Variáveis } & Média & Mediana \\
\hline $\begin{array}{l}\text { Podemos lidar rapidamente com os conflitos no processo de tomada de decisão } \\
\text { estratégico. CDA1 }\end{array}$ & $\mathbf{2 , 8 4 2}$ & $\mathbf{3}$ \\
\begin{tabular}{|l|c|} 
Em muitas circunstâncias, podemos tomar decisões em tempo hábil para lidar com \\
problemas estratégicos. CDA2
\end{tabular} & $\mathbf{3 , 0 5 0}$ & $\mathbf{3}$ \\
\begin{tabular}{|l|c|} 
Podemos remediar rapidamente os problemas com estudantes insatisfeitos. CDA3 \\
$\begin{array}{l}\text { Podemos replanejar os recursos a tempo de resolver um problema de mudança ambiental. } \\
\text { CDA4 }\end{array}$
\end{tabular} & $\mathbf{2 , 6 3 2}$ & $\mathbf{3}$ \\
\hline
\end{tabular}

Fonte: Dados da pesquisa (2019).

Tomar decisões em tempo hábil para lidar com problemas estratégicos e remediar rapidamente os problemas com estudantes insatisfeitos, obtiveram médias 3,050 e 3158, que indicam a boa percepção dos gestores institucionais, no que se refere a essas variáveis. A rapidez para lidar com os conflitos no processo de tomada de decisão estratégica e o replanejamento dos recursos a tempo de resolver um problema de mudança ambiental, indicaram médias 2,842 e 2,632, o que ressalta a dificuldade em tomar decisões antecipadamente, principalmente no que se refere a recursos financeiros. Thoenig (2016), enfatiza que a extensão em que as prioridades, as preferências e as iniciativas tomadas dentro da instituição ao longo do tempo e em seu ambiente se harmonizam depende muito de quão bem a linha programática que a organização cria se encaixa, assim, organizando recursos adequados para serem alocados para esses fins (Thoenig, 2016).

Tabela 3: Capacidade de Alterar a Implementação

\begin{tabular}{l|c|c}
\hline \multicolumn{1}{c|}{ Variáveis } & Média & Mediana \\
\hline Nossas mudanças estratégicas podem ser eficientemente realizadas. - CAI1 & $\mathbf{3 , 3 1 6}$ & $\mathbf{3}$ \\
\hline Existe boa cooperação entre diferentes setores/áreas. - CAI2 & $\mathbf{2 , 9 0 0}$ & $\mathbf{3}$ \\
\hline Nós ajudamos mutuamente na implementação de mudanças estratégicas. - CAI3 & $\mathbf{2 , 7 8 9}$ & $\mathbf{3}$ \\
\hline
\end{tabular}

UFAMBR, Manaus, v. 3, n. 1, art. 3, pp. 39-53, janeiro-junho, 2021 http://www.periodicos.ufam.edu.br/ufambr 
Capacidades Dinâmicas, Dinamismo Ambiental e Vantagem Competitiva: Evidências de uma Instituição Comunitária de Ensino Superior

\begin{tabular}{|l|c|c|}
\hline Nós temos um sistema próprio de controle. - CAI5 & $\mathbf{3 , 2 1 1}$ & $\mathbf{3}$ \\
\hline Podemos melhorar eficientemente a mudança na implementação estratégica. - CAI6 & $\mathbf{3 , 6 8 4}$ & $\mathbf{4}$ \\
\hline
\end{tabular}

Fonte: Dados da pesquisa (2019).

A eficiência nas mudanças estratégicas, o sistema próprio de controle e a melhoria eficiente na mudança da implementação estratégica, possuem médias de 3,316, 3,211 e 3,684, o que ressalta indicadores de boa percepção nestas variáveis de pesquisa. A boa cooperação entre diferentes setores ou áreas e a ajuda mútua na implementação de mudanças estratégicas, não possuem bons indicadores. Estas variáveis obtiveram médias 2,900 e 2,789, que significam a necessidade de implementar uma cultura de boa cooperação e ajuda entre os colaboradores da universidade. Em seus estudos, Frølich (2019), relata que a capacidade estratégica é um conceito que se relaciona com os processos associados à formação, organização e implementação de estratégias em universidades e faculdades. Portanto, a dinâmica de implementação molda as capacidades de formulação de estratégias (Thoenig, 2016).

Tabela 4: Vantagem Competitiva

\begin{tabular}{l|c|c|}
\hline \multicolumn{1}{c|}{ Variáveis } & Média & Mediana \\
\hline $\begin{array}{l}\text { Em comparação com os nossos concorrentes, temos maior taxa de crescimento do lucro. } \\
\text { - VC1 }\end{array}$ & $\mathbf{2 , 6 8 4}$ & $\mathbf{3}$ \\
\hline $\begin{array}{l}\text { Em comparação com os nossos concorrentes, temos maior taxa de crescimento de receita } \\
\text { de vendas. - VC2 }\end{array}$ & $\mathbf{2 , 6 3 2}$ & $\mathbf{3}$ \\
\hline $\begin{array}{l}\text { Em comparação com os nossos concorrentes, temos custos operacionais mais baixos. - } \\
\text { VC3 }\end{array}$ & $\mathbf{2 , 2 6 3}$ & $\mathbf{3}$ \\
\hline $\begin{array}{l}\text { Em comparação com os nossos concorrentes, temos melhor produto e qualidade de } \\
\text { serviço. - VC4 }\end{array}$ & $\mathbf{4 , 0 5 0}$ & $\mathbf{4}$ \\
\hline $\begin{array}{l}\text { Em comparação com os nossos concorrentes, temos parcela de mercado cada vez maior. } \\
\text { - VC5 }\end{array}$ & $\mathbf{2 , 3 5 0}$ & $\mathbf{2 , 5}$ \\
\hline $\begin{array}{l}\text { Egressos que trabalham na área de formação. - VC6 } \\
\text { Estudantes mais participativos na comunidade. - VC7 }\end{array}$ & $\mathbf{3 , 9 0 0}$ & $\mathbf{4}$ \\
\hline
\end{tabular}

Fonte: Dados da pesquisa (2019).

Maior taxa de crescimento do lucro e da receita de vendas, custos operacionais mais baixos e parcela de mercado cada vez maior em comparação com os concorrentes, obtiveram índices entre 2,263 e 2,264, ressaltando que diante da percepção dos gestores, a instituição não possui vantagem competitiva nestes quesitos. As variáveis de melhor produto e qualidade de serviço, egressos que trabalham na área de formação e estudantes participativos na comunidade, resultaram em indicadores elevados, sendo 4,050, 3,900 e 3,750, fato este, que enfatiza a elevada qualidade no ensino de acordo com os gestores da universidade. Em seus estudos, Al Shobaki \& Abu-Naser (2017), relatam que a vantagem competitiva das instituições de ensino decorre do impacto sobre o nível de cada aluno, funcionário e da própria instituição. Portanto, existe uma forte correlação entre o nível de exercício de estratégias de excelência na educação e a conquista de instituições de ensino superior para a vantagem competitiva sustentável.

\section{Tabela 5: Percepção de Dinamismo Ambiental}

UFAMBR, Manaus, v. 3, n. 1, art. 3, pp. 39-53, janeiro-junho, 2021 http://www.periodicos.ufam.edu.br/ufambr 
Capacidades Dinâmicas, Dinamismo Ambiental e Vantagem Competitiva: Evidências de uma Instituição Comunitária de Ensino Superior

\begin{tabular}{l|l|l}
\hline \multicolumn{1}{c|}{ Variáveis } & Média & Mediana \\
\hline O produto ou serviço em nossa instituição é atualizado no tempo adequado. - PDA1 & $\mathbf{3 , 0 5 0}$ & $\mathbf{3}$ \\
\hline As ações dos concorrentes são fáceis de prever. - PDA2 & $\mathbf{2 , 6 0 0}$ & $\mathbf{3}$ \\
\hline A tecnologia em nossa instituição progride rapidamente - PDA3 & $\mathbf{4 , 0 5 0}$ & $\mathbf{4}$ \\
\hline Conseguimos prever a mudança das necessidades dos alunos. - PDA4 & $\mathbf{3 , 1 0 5}$ & $\mathbf{3}$ \\
\hline
\end{tabular}

Fonte: Dados da pesquisa (2019).

A atualização do produto ou serviço no tempo adequado, a rápida progressão tecnológica e a previsão de mudança das necessidades dos alunos, obtiveram médias 3,050, 4,050 e 3,105, indicando, na percepção dos gestores, que a instituição possui pontos fortes nestes quesitos. A fácil previsão das ações dos concorrentes, não teve índice elevado. Com média de 2,600 a instituição indica a necessidade de fortalecimento nesta variável de pesquisa. Jansen et al. (2006) ressalta que o dinamismo ambiental é caracterizado por mudanças tecnológicas, variações nas preferências do cliente, mudanças na demanda de produtos e imprevisibilidade da mudança. Portanto, devido ao alto índice de mudança no ambiente que estão enfrentando, as organizações geralmente têm recursos muito limitados de informações e, portanto, previsões ruins em termos do que levará ao sucesso (Hou et al., 2019).

\section{CONCLUSÃO}

O estudo objetivou evidenciar relação entre as capacidades dinâmicas, dinamismo ambiental e vantagem competitiva em uma instituição de ensino superior comunitária. Para tanto, foi aplicado um questionário para a coleta de dados que demonstrou um conjunto de informações acerca do foco priorizado na realidade desta instituição de ensino superior, conforme delineado nas seções anteriores. Desta forma, foi possível responder à questão de estudo.

As capacidades dinâmicas, o dinamismo ambiental e a vantagem competitiva, são critérios fundamentais para o desempenho de uma organização. Cada vez mais evidencia-se a necessidade de mudanças rápidas e assertivas nas estratégias empresariais, e o mesmo se aplica às instituições de ensino superior. $O$ processo de tornar-se uma organização reflete uma dinâmica de racionalização mais ampla, obrigando a universidade a adquirir as características de uma entidade organizacional integrada e de atuação estratégica (Ramirez, 2006).

Por meio da análise abordada, as relações diretas das capacidades dinâmicas, dinamismo ambiental e vantagem competitiva, mostram a necessidade que uma instituição de ensino superior tem em possuir senso de capacidade estratégica, capacidade de decisão antecipada, capacidade de alterar a implementação das estratégias, vantagem competitiva em relação aos seus concorrentes e percepção de dinamismo ambiental, para que conquiste níveis elevados de sustentação na gestão estratégica.

Para finalizar, a título de recomendação para trabalhos futuros, propõe-se que sejam investigados outros critérios de gestão em relação à estratégia no âmbito das universidades. Caberia, também, replicar pesquisa assemelhada em outras regiões do país, com a finalidade de confrontar as realidades apuradas nestes contextos diversos.

\section{REFERÊNCIAS}

UFAMBR, Manaus, v. 3, n. 1, art. 3, pp. 39-53, janeiro-junho, 2021 http://www.periodicos.ufam.edu.br/ufambr 
Capacidades Dinâmicas, Dinamismo Ambiental e Vantagem Competitiva: Evidências de uma Instituição Comunitária de Ensino Superior

Al Shobaki, M. J., \& Abu-Naser, S. S. (2017). The Role of the Practice of Excellence Strategies in Education to Achieve Sustainable Competitive Advantage to Institutions of Higher Education-Faculty of Engineering and Information Technology at Al-Azhar University in Gaza a Model.

Ansoff, H. I. (1987). The emerging paradigm of strategic behavior. Strategic management journal, 8(6), 501-515.

Anwar, M. (2018). Business model innovation and SMEs performance-does competitive advantage mediate?. International Journal of Innovation Management, 22(07), 1850057.

Aslan, M. (2021). Estrutura organizacional revisitada. Business \& Management Studies: An International Journal , 9 (1), 282-294.

Borges-Andrade, J. E., Coelho Jr, F. A., \& Queiroga, F. (2006). Pesquisa sobre micro comportamento organizacional no Brasil: o estado da arte. In Anais eletrônicos do II Congresso Brasileiro de Psicologia Organizacional e do Trabalho. Brasília: SBPOT.

Brandenburger, A. M., \& Stuart Jr, H. W. (1996). Value-based business strategy. Journal of economics \& management strategy, 5(1), 5-24.

Chan, HK, Yee, RW, Dai, J., \& Lim, MK (2016). O efeito moderador do dinamismo ambiental na inovação e no desempenho de produtos verdes. International Journal of Production Economics , 181 , 384-391.

Chandler, A. D. (1990). Strategy and structure: Chapters in the history of the industrial enterprise (Vol. 120). MIT press.

Chang, S. J., \& Park, S. H. (2012). Winning strategies in China: Competitive dynamics between MNCs and local firms. Long Range Planning, 45(1), 1-15.

Chiavenato, I. (2010). Gestão de pessoas. 3. Ed. Rio de Janeiro, RJ: Campus.

Chirico, F., \& Bau', M. (2014). Is the family an "asset" or "liability" for firm performance? The moderating role of environmental dynamism. Journal of Small Business Management, 52(2), 210-225.

Covin, J. G., \& Slevin, D. P. (1991). A conceptual model of entrepreneurship as firm behavior. Entrepreneurship theory and practice, 16(1), 7-26.

Coutinho, L., \& Ferraz, J. C. (1994). Estudo da competitividade da indústria brasileira. Campinas: Papirus.

Cui, L., Meyer, K. E., \& Hu, H. W. (2014). What drives firms' intent to seek strategic assets by foreign direct investment? A study of emerging economy firms. Journal of World Business, 49(4), 488-501.

D'Aveni, R. A., Dagnino, G. B., \& Smith, K. G. (2010). The age of temporary advantage. Strategic management journal, 31(13), 1371-1385.

D'Aveni, RA, \& Gunther, R. (1994). Gerenciando a dinâmica de manobras estratégicas. New York .

Demil, B., Lecocq, X., Ricart, J. E., \& Zott, C. (2015). Introduction to the SEJ special issue on business models: business models within the domain of strategic entrepreneurship.

Eisenhardt, K. M., \& Martin, J. A. (2000). Dynamic capabilities: what are they?. Strategic management journal, 21(10-11), 1105-1121.

Eroglu, C., \& Hofer, C. (2014). The effect of environmental dynamism on returns to inventory leanness. Journal of Operations Management, 32(6), 347-356.

Ferrier, WJ, Smith, KG e Grimm, CM (1999). O papel da ação competitiva na erosão da participação de mercado e destronamento da indústria: um estudo dos líderes e desafiadores da indústria. Academy of management journal , 42 (4), 372-388.

Figueiredo, P. (2009). Gestão da inovação: conceitos, métricas e experiências de empresas no Brasil. Livros Técnicos e Científicos. 
Capacidades Dinâmicas, Dinamismo Ambiental e Vantagem Competitiva: Evidências de uma Instituição Comunitária de Ensino Superior

Frølich, N., Christensen, T., \& Stensaker, B. (2019). Strengthening the strategic capacity of public universities: The role of internal governance models. Public Policy and Administration, 34(4), 475-493.

Gadiesh, Orit; Leung, Philip; Vestring, Till (2007). The battle for China's good-enough market. Harvard Business Review, v. 85, n. 9, p. 80.

Hair, J., Babin, B., Money, A., \& Samouel, P. (2005). Fundamentos de métodos de pesquisa em administração. Bookman Companhia Ed.

Håkansson, H., \& Snehota, I. (1989). No business is an island: The network concept of business strategy. Scandinavian journal of management, 5(3), 187-200.

Halawi, L. A., Aronson, J. E., \& McCarthy, R. V. (2005). Resource-based view of knowledge management for competitive advantage. The electronic journal of knowledge management, 3(2), 75.

Helfat, C. E., Finkelstein, S., Mitchell, W., Peteraf, M., Singh, H., Teece, D., \& Winter, S. G. (2009). Dynamic capabilities: Understanding strategic change in organizations. John Wiley \& Sons.

Hofer, C. W., \& Schendel, D. (1980). Strategy formulation: Analytical concepts. West Publ..

Hou, B., Hong, J., Zhu, K., \& Zhou, Y. (2019). Paternalistic leadership and innovation: the moderating effect of environmental dynamism. European Journal of Innovation Management.

Jansen, J. J., Van Den Bosch, F. A., \& Volberda, H. W. (2006). Exploratory innovation, exploitative innovation, and performance: Effects of organizational antecedents and environmental moderators. Management science, 52(11), 1661-1674.

Marconi, M. D. A., \& Lakatos, E. M. (2003). Fundamentos de metodologia científica. 5. ed.São Paulo: Atlas.

Li, DY, \& Liu, J. (2014). Capacidades dinâmicas, dinamismo ambiental e vantagem competitiva: evidências da China. Journal of business research , 67 (1), 2793-2799.

Mahdi, OR, Nassar, IA e Almsafir, MK (2019). Processos de gestão do conhecimento e vantagem competitiva sustentável: um exame empírico em universidades privadas. Journal of Business Research , 94 , 320-334.

Martins, W., \& Escrita, A. P. (2001). história do livro, da imprensa e da biblioteca.

Matthyssens, P., \& Vandenbempt, K. (2008). Mudando de ofertas básicas para soluções de valor agregado: Estratégias, barreiras e alinhamento. Gestão de Marketing Industrial , 37 (3), 316-328.

Mintzberg, H., \& McHugh, A. (1985). Formação de estratégia em uma adhocracia. Ciências administrativas trimestralmente, 160-197.

Moore, S. (1995). Making sense of strategic management: towards a constructive guide. Management Decision.

Oliveira, Djalma de Pinho Rebouças de. (2001). Planejamento estratégico: conceito, metodologia e práticas. 15.ed. São Paulo: Atlas.

Peteraf, M. A., \& Barney, J. B. (2003). Unraveling the resource-based tangle. Managerial and decision economics, 24(4), 309-323.

Porter, M. E. (1999). Competição: estratégias competitivas essenciais. Gulf Professional Publishing.

Possas, M. L. (1999). Demanda efetiva, investimento e dinâmica. Revista Economia Comtemporânea, Rio de Janeiro, 3(2).

Raduan, C. R., Jegak, U., Haslinda, A., \& Alimin, I. I. (2009). Management, strategic management theories and the linkage with organizational competitive advantage from the resource-based view. European Journal of Social Sciences, 11(3), 402-418. 
Capacidades Dinâmicas, Dinamismo Ambiental e Vantagem Competitiva: Evidências de uma Instituição Comunitária de Ensino Superior

Ramirez, F. (2006). Growing commonalities and persistent differences in higher education: Universities between global models and national legacies. The new institutionalism in education, 123-142.

Richardson, R. J. (1999). Pesquisa social: métodos e técnicas. São Paulo: Atlas. Rios, TA. Ética e Competência.

Rothaermel, FT (2016). Vantagem Competitiva em Indústrias de Tecnologia Intensiva (Vol. 26, pp. 233-256). Emerald Publishing Ltd.

Salunke, S., Weerawardena, J., \& McColl-Kennedy, J. R. (2019). The central role of knowledge integration capability in service innovation-based competitive strategy. Industrial Marketing Management, 76, 144-156.

Sausen, J. O. (2002). Adaptação estratégica organizacional.

Schiuma, G., Carlucci, D., \& Lerro, A. (2012). Managing knowledge processes for value creation. Vine.

Schoemaker, P. J., Heaton, S., \& Teece, D. (2018). Innovation, dynamic capabilities, and leadership. California Management Review, 61(1), 15-42.

Shane, S., \& Venkataraman, S. (2000). The promise of entrepreneurship as a field of research. Academy of management review, 25(1), 217-226.

Smith, K. G., Ferrier, W. J., \& Ndofor, H. (2001). Competitive dynamics research: Critique and future directions. Handbook of strategic management, 315, 361.

Story, V. M., Boso, N., \& Cadogan, J. W. (2015). The form of relationship between firm-level product innovativeness and new product performance in developed and emerging markets. Journal of Product Innovation Management, 32(1), 45-64.

Teece, D. J. (2018). Business models and dynamic capabilities. Long range planning, 51(1), 40-49.

Teece, D. J. (2010). Business models, business strategy and innovation. Long range planning, 43(2-3), 172-194.

Teece, D. J., Pisano, G., \& Shuen, A. (1997). Dynamic capabilities and strategic management. Strategic management journal, 18(7), 509-533.

Thoenig, J. C., \& Paradeise, C. (2016). Strategic capacity and organisational capabilities: A challenge for universities. Minerva, 54(3), 293-324.

Tregoe, B. B., \& Zimmerman, J. W. (1980). Top management strategy: What it is and how to make it work.

Wamba, S. F., Dubey, R., Gunasekaran, A., \& Akter, S. (2020). The performance effects of big data analytics and supply chain ambidexterity: The moderating effect of environmental dynamism. International Journal of Production Economics, 222, 107498.

Yu, H., Wang, X., Wang, G., \& Zeng, X. (2020). An active three-way clustering method via low-rank Wang, X., Sun, J., Tian, L., Guo, W., \& Gu, T. (2020). Dinamismo ambiental e inovação cooperativa: o papel moderador da propriedade estatal e do desenvolvimento institucional. The Journal of Technology Transfer, 1-32.

Zahra, S. A. (1993). Environment, corporate entrepreneurship, and financial performance: A taxonomic approach. Journal of business venturing, 8(4), 319-340.

Zahra, S. A., \& Covin, J. G. (1993). Business strategy, technology policy and firm performance. Strategic management journal, 14(6), 451-478.

Zhang, F., Chen, J., \& Zhu, L. (2021). How Does Environmental Dynamism Impact Green Process Innovation? A Supply Chain Cooperation Perspective. IEEE Transactions on Engineering Management.

Zhang, F., Chen, J., Zhu, L., \& Liu, L. (2021). Does resource slack promote or constrain firm environmental management investment? Moderating roles of technology sources. Total Quality Management \& Business Excellence, 1-24. 